\title{
Intrinsic localisation in the dynamics of a Josephson-junction ladder
}

\author{
L. M. Floría ${ }^{1,2}$, J. L. Marín ${ }^{1,2}$, P. J. Martínez ${ }^{1}$, F. FAlO $^{1}$ \\ and S. AuBrY ${ }^{2}$ \\ 1 Departamento de Fúsica de la Materia Condensada \\ Instituto de Ciencia de Materiales de Aragón, CSIC \\ Universidad de Zaragoza - 50009 Zaragoza, Spain \\ 2 Laboratoire Léon Brillouin (CEA-CNRS), CE Saclay \\ 91191 Gif-sur-Yvette Cedex, France
}

(received 13 June 1996; accepted in final form 22 October 1996)

PACS. 74.50+r - Proximity effects, weak links, tunneling phenomena, and Josephson effects.

PACS. 03.20+i - Classical mechanics of discrete systems: general mathematical aspects.

PACS. 85.25Cp - Josephson devices.

\begin{abstract}
We investigate some aspects of the general problem of nonlinear effects on the operation of an anisotropic ladder of Josephson junctions with injected ac currents. We predict the existence of attracting time-periodic spatially localised modes, for some ranges of junction characteristic parameters. These elementary dynamical excitations are of two different types, associated to oscillatory and rotating motion of a few superconducting islands phases, respectively, revealing a dynamical mechanism of creation of vortex-antivortex pairs. These results are physical applications of recent advances in the theory of nonlinear dynamics of discrete macroscopic systems. Their experimental confirmation would probe the physical relevance of localisation in Josephson-junction devices.
\end{abstract}

Josephson-junction devices are, among many other important applications, the way the voltage standard is established. Not surprisingly, experimental and theoretical understanding of their operation has been for years, and still is, an active research field of both fundamental and practical importance. The theory of the Josephson effect contains essentially nonlinear aspects and its predictions can be seen to be closely related to the general physics of a forced and damped mathematical pendulum. On the other hand, recent impressive developments of nonlinear science provide new concepts and perspectives which are penetrating many areas of physical research, shedding new light and raising new questions about an increasing number of physical systems. It is in this direction that the work presented below is framed.

We analyse here the dynamics of a Josephson-junction ladder with injected ac currents and find the existence of attracting time-periodic solutions whose energy is exponentially localised. They can be classified in two groups: i) oscillator localised modes, in which the amplitude of the superconducting phase oscillation is exponentially localised, and ii) rotor localised modes, 


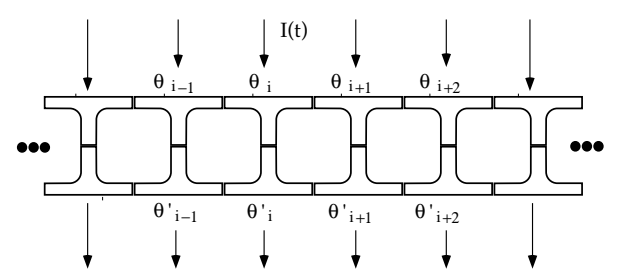

Fig. 1. - Schematic picture of the JJ ladder.

where the phases of a pair of superconducting islands rotate while the rest perform forced oscillations. The physical consequences of their existence is analysed afterwards.

The existence and characterisation of intrinsically localised modes in (Hamiltonian and non-Hamiltonian) discrete arrays of nonlinear oscillators has been anticipated by different analytical and numerical approximations [1]-[3] and has recently been given support by new theorems of nonlinear dynamical systems theory [4] exploiting the ideas of anticontinuous limit [5], [6]. Many issues concerning their stability, movability, etc. are still open questions in spite of some recent progress [7], [8]. These localised modes (also known as discrete breathers) are intrinsic, in the sense that they occur even if the system is homogeneous (no impurities or disorder are present), so that the localisation is due to nonlinearity.

Superconducting arrays consist of superconducting grains (islands) embedded in a normal or insulating matrix, linked together by Josephson or proximity effect couplings. The superconducting order parameter (condensate wave function) of the island located at position $\mathbf{x}$ is $\Psi(\mathbf{x})=|\Psi(\mathbf{x})| \exp [i \theta(\mathbf{x})]$. We will consider arrays with a ladder geometry (see fig. 1), whose ground-state properties are now well established [9], [10]. The basic equations for the operation of a Josephson-junction array biased by injected currents $I(t)=I \cos (\omega t)$, in the framework of the resistively and capacitively shunted junction (RCSJ) approach [11], [12], neglecting inductive effects and incorporating capacitive effects (through an inertial behaviour of the superconducting phases) are

$$
\ddot{\theta}(\mathbf{x})=\sum_{\delta}\left[J_{\delta} \sin (\theta(\mathbf{x}+\delta)-\theta(\mathbf{x}))+\epsilon_{\delta}(\dot{\theta}(\mathbf{x}+\delta)-\dot{\theta}(\mathbf{x}))\right] \mp I(t),
$$

where $\delta$ are nearest-neighbour lattice vectors. $J_{\delta}$ is the Josephson coupling (critical current, $\left.I_{\mathrm{c}, \delta}\right)$ of the junction attached to the link $\delta$ and $\epsilon_{\delta}$ is related to the resistive effects from the contribution of normal electrons; both are junction characteristics. As shown in fig. 1, the currents $I(t)$ are injected into the islands in the upper row and extracted from those in the lower row. More specifically, we will assume that the junction characteristics are $\left(J_{x}, \epsilon_{x}\right)$ for junctions in the horizontal rows, and $\left(J_{y}, \epsilon_{y}\right)$ for junctions which couple islands in different rows. Also, $\theta_{i}$ and $\theta_{i}^{\prime}$ will denote, respectively, the phases of upper and lower islands at site $i$ in the ladder.

With the change of variables (center of mass and relative coordinates) $\chi_{i}=\frac{1}{2}\left(\theta_{i}+\theta_{i}^{\prime}\right)$, $\phi_{i}=\frac{1}{2}\left(\theta_{i}-\theta_{i}^{\prime}\right)$, eq. (1) can be written as

$$
\begin{gathered}
\ddot{\chi}_{i}=J_{x}\left[\sin \left(\chi_{i+1}-\chi_{i}\right) \cos \left(\phi_{i+1}-\phi_{i}\right)+\sin \left(\chi_{i-1}-\chi_{i}\right) \cos \left(\phi_{i-1}-\phi_{i}\right)\right]+ \\
+\epsilon_{x}\left(\dot{\chi}_{i+1}+\dot{\chi}_{i-1}-2 \dot{\chi}_{i}\right) \\
\ddot{\phi}_{i}=J_{x}\left[\cos \left(\chi_{i+1}-\chi_{i}\right) \sin \left(\phi_{i+1}-\phi_{i}\right)+\cos \left(\chi_{i-1}-\chi_{i}\right) \sin \left(\phi_{i-1}-\phi_{i}\right)\right]+ \\
\quad+\epsilon_{x}\left(\dot{\phi}_{i+1}+\dot{\phi}_{i-1}-2 \dot{\phi}_{i}\right)-J_{y} \sin \left(2 \phi_{i}\right)-2 \epsilon_{y} \dot{\phi}_{i}-I(t) .
\end{gathered}
$$




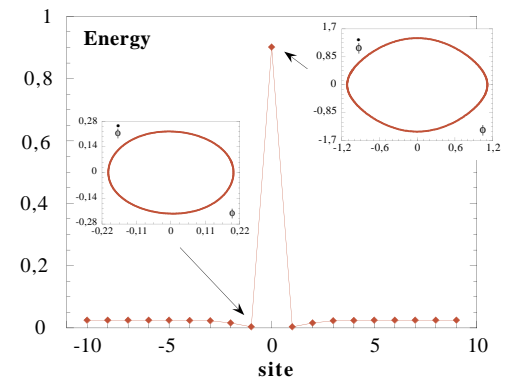

Fig. 2. - Average energy (kinetic + Josephson) profile of an oscillator localised mode $\left(J_{y}=0.5, J_{x}=\right.$ $0.04, \epsilon_{y}=\epsilon_{x}=0.01, I=0.1, \omega=0.8$. See text above eq. (1)). The insets show the phase space trajectories of the central and nearest-neighbour phases (note the different scales).

With uniform initial conditions in the center-of-mass coordinates and momenta, i.e. $\chi_{i}, \dot{\chi}_{i}$ independent of $i,(2 a)$ have the solutions $\chi_{i}(t)=\Omega t+\alpha$, for all $i$. In this case, the dynamics reduces to the following equations of motion $\left(^{1}\right)$ for the phase half-differences, $\phi_{i}$ :

$$
\ddot{\phi}_{i}=\tilde{\eta}\left[\sin \left(\phi_{i+1}-\phi_{i}\right)+\sin \left(\phi_{i-1}-\phi_{i}\right)\right]+\tilde{\epsilon}_{x}\left(\dot{\phi}_{i+1}+\dot{\phi}_{i-1}-2 \dot{\phi}_{i}\right)-\sin \left(2 \phi_{i}\right)-2 \tilde{\epsilon}_{y} \dot{\phi}_{i}-\tilde{I}(t) .
$$

Following the idea of the above-mentioned theorems [4] one can construct a proof of the existence of time-periodic solutions to (3) with exponential localization; technical details will appear elsewhere, though the essence of the argument is implicit in [4], [8]. We will concentrate here on the physical characterisation of this type of solution for the Josephson-junction ladder system.

In order to explicitly compute the localised solutions (both oscillator and rotor localised modes) we have used the numerical procedure developed in [8]. Based on rigorous mathematical foundations and incorporating well-established numerical methods of fixed-point iteration and integration of ODEs, this method provides the exponentially localised solution as an attractor of a functional operator in certain space of sequences of functions. The solution can then be given as the initial condition to a direct simulation of the full system of equations of motion (2) in order to check its attracting character, robustness against different types of fluctuations, etc.

Figure 2 shows the energy profile of an oscillator localised mode in the ladder for a particular choice of the parameter values. In the inset of fig. 2 we show the closed trajectories in the phase space $(\phi, \dot{\phi})$ of the phase half-differences across vertical junctions. Voltage profiles across horizontal and vertical junctions are shown in fig. 3. The high precision of the numerical method of Marín and Aubry allows for a precise Floquet analysis of the linear stability of these solutions. We are currently carrying out an exhaustive exploration in parameter space of this issue, in order to determine the instability mechanisms. From our preliminary results we can conclude in favour of the existence of wide zones for linear stability of the oscillator localised modes, mostly for low values of the anisotropy $\tilde{\eta}$. This is what should be expected, for localisation is naturally favoured by low values of the horizontal coupling between

$\left({ }^{1}\right)$ The unit time from eq. (3) onwards is $\tau=\hbar\left(8 E_{\mathrm{C}} E_{\mathrm{J}}\right)^{-1 / 2}$. The dimensionless anisotropy parameter is $\tilde{\eta}=J_{x} / J_{y}=I_{\mathrm{c}, x} / I_{\mathrm{c}, y}$, and the dissipative couplings $(\alpha=x, y) \tilde{\epsilon}_{\alpha}=\left(\hbar / e^{2}\right) R^{-1}\left(E_{\mathrm{C}} / E_{\mathrm{J}_{\alpha}}\right)^{1 / 2}$; here $E_{\mathrm{C}}=e^{2} /(2 C)$ and $E_{\mathrm{J}}=\hbar I_{\mathrm{c}} /(2 e)$ are the charging and Josephson energies, respectively. Finally, $\tilde{I}(t)$ is measured in units of $I_{\mathrm{c}, y}$. 

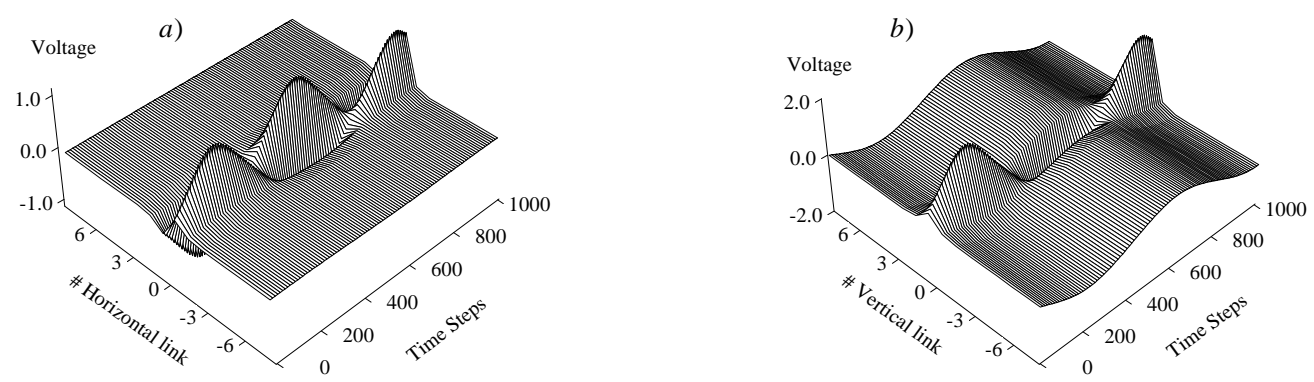

Fig. 3. - Voltage profiles (in units of $\hbar /(2 e \tau)$, see footnote $\left({ }^{1}\right)$ ) across horizontal $(a)$ ) and vertical $(b)$ ) links, for the oscillator localised mode shown in the previous figure. The integration time step in this and the next figures is 0.01 .

superconducting phases.

We have checked the attracting character of these solutions by direct numerical integration of the original equations of motion (2). For arbitrary, small enough, perturbations of the localised solution, the perturbed trajectories are observed to tend to the unperturbed localised mode, confirming that it is an attractor of the dynamics. This suggests a nonnegligible chance of observing these localised modes in experiments, for an appropriate range of fabricated junction characteristics.

In analogy with [4] we also predict the existence of arrays of modes localised at different sites. We have also computed these multi-mode solutions and confirmed their attracting character. In fact, in the range of low values of $\left(J_{x}, \epsilon_{x}\right)$, for a wide set of arbitrary initial conditions, the attractor reached after (usually long) transients is generically a (glassy-like) array of localised modes.

The second class of localised solutions, the rotor localised mode, differs from the previous one in that at the site of energy localisation, the superconducting phases rotate, while the rest remain oscillating (see fig. 4). For a Hamiltonian array of pendulums with periodic coupling, this type of localised mode was first found explicitly by Takeno and Peyrard [13], and its existence was implicitly envisaged in the theorems of [4]. Note that in the Josephson-junction ladder there are additional external forcing, damping and momenta coupling.

The most significant feature of a rotor localised mode concerns the vorticity profile of the

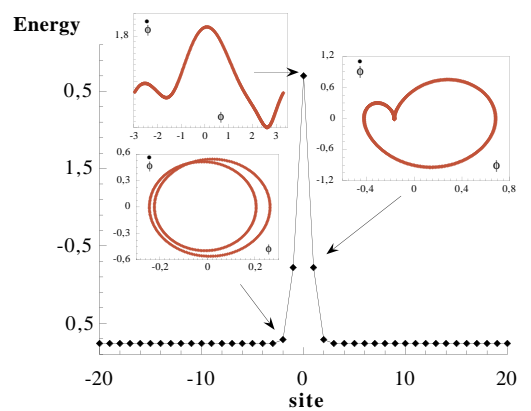

Fig. 4. - Average energy profile of a rotor localised mode for the parameter values $J_{y}=0.5, J_{x}=0.18$, $\epsilon_{x}=\epsilon_{y}=0.01, I=0.3, \omega=1.5$. The insets show the phase space trajectories of the central and neighbouring phases. 


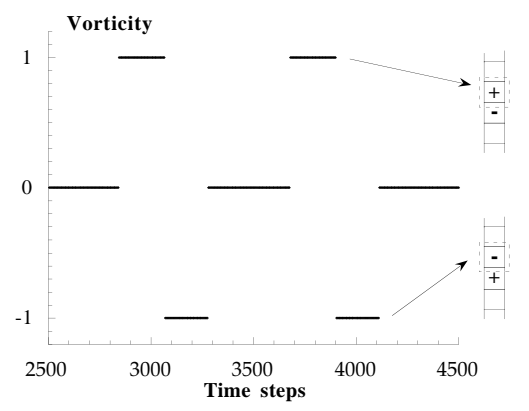

Fig. 5. - Time evolution of the vorticity of one of the central plaquettes for the rotor localised mode in the previous figure.

ladder. The number of fluxoid quanta (vortices) $n_{i}$ associated to the $i$-th plaquette in the ladder is defined in terms of the circulation around the plaquette of the superconducting phase gradient. In the absence of external magnetic field, which is the case we analyse here, one has

$$
\oint_{\Gamma_{i}} \nabla \theta \cdot \mathbf{d} l=2 \pi n_{i}
$$

where the contour integral is performed around a loop $\Gamma_{i}$ enclosing the $i$-th plaquette. Figure 5 shows the time-dependent vorticity profile of the ladder for a rotor localised mode computed with the method of Marín and Aubry. The excitation energy of this mode (which is a microscopic quantity due to the localised character of the solution) manifests itself through the intermittent creation and subsequent annihilation of a pair of fluxoid quanta of opposite sign (vortex-antivortex) located in the central plaquettes.

Though we have considered the situation in which no external magnetic field is present, the existence of energy localisation persists under magnetic fields. Interesting phenomena are then expected to appear as a result of the interplay between commensurability (and the associated defectibility [10]) and localisation. In any case, we should recall that most applications of Josephson-junction-array-based devices rely on the coherent synchronous motion of the phases; what we have shown here constitutes a new phenomenon which could destroy this coherence (the eventual unbinding of pairs and subsequent vortex motion), and is, therefore, something to be avoided in such devices, or perhaps, due to the robustness and generality of this novel effect, something to be turned to our advantage in possible new applications (e.g., photodetection).

From what experimental feasibility is concerned with, the RCSJ model is usually considered to give a sensible description for the classical junction regime, $\left(E_{\mathrm{C}} / E_{\mathrm{J}}\right) \ll 1$ (see footnote $\left(^{1}\right)$ ), while the underdamped regime of the dynamics should be fine for low values of $\tilde{\epsilon}_{\delta}$. Both conditions can be easily fulfilled in tunnel junctions (see, for example, [14]). The parameters shown in the figures would correspond, assuming for example $R=10 \mathrm{k} \Omega$ and $I_{\mathrm{c}}=0.03 \mu \mathrm{A}$, to $\left(E_{\mathrm{C}} / E_{\mathrm{J}}\right) \approx 10^{-3}$ and $\omega \approx 9 \mathrm{GHz}$, just to give an idea about feasibility matters.

Our results point toward the utmost importance of experimental work on Josephsonjunction ladders, as a benchmark system for nonlinear concepts, in the firm belief that the interaction between theory and experiment is the way toward serious advances in nonlinear condensed-matter physics.

$$
* * *
$$

We are indebted to J. J. MAZO and R. S. MACKAY for the critical reading of the 
manuscript. We acknowledge financial support of the European Union, through the Network (CHRX-CT93-0331), and CICYT (PB92-0361), Spain.

\section{REFERENCES}

[1] Eilbeck C., Lomdahl P. S. and Scott A. C., Physica D, 16 (1983) 318.

[2] Sievers A. J. and Takeno S., Phys. Rev. Lett., 61 (1988) 970; Takeno S., Kisoda K. and Sievers A. J., Prog. Theor. Phys. Suppl., 94 (1988) 242.

[3] Dauxois T., Peyrard M. and Willis C. R., Physica D, 57 (1992) 267; Dauxois T. and Peyrard M., Phys. Rev. Lett., 70 (1993) 3935.

[4] Mackay R. S. and Aubry S., Nonlinearity, 7 (1994) 1623.

[5] Aubry S., Physica D, 86 (1995) 284.

[6] Mackay R. S., Physica D, 86 (1995) 122.

[7] Flach S. and Willis C. R., Phys. Rev. Lett., 72 (1994) 1777.

[8] Marín J. L. and Aubry S., to be published in Nonlinearity; Aubry S., Proceedings of the Workshop on Lattice Dynamics, to be published in Physica D.

[9] Kardar M., Phys. Rev. B, 30 (1984) 6368; Kardar M., Phys. Rev. B, 33 (1986) 3125.

[10] Mazo J. J., Falo F. and Floría L. M., Phys. Rev. B, 52 (1995) 10433; Floría L. M. and Mazo J. J., to be published in Adv. Phys. (1996).

[11] Tinkham M., Introduction to Superconductivity, second edition (McGraw-Hill, New York, N.Y.) 1996.

[12] Falo F., Bishop A. R. and Lomdahl P. S., Phys. Rev. B, 41 (1990) 10983; Wang Z. Q. and Stroud D., Phys. Rev. B, 44 (1991) 9643.

[13] Takeno S. and Peyrard M., preprint (1995).

[14] Van der Zant H. S. J., Fritschy F. C., Orlando T. P. and Mooij J. E., Phys. Rev. B, 47 (1993) 295. 